\title{
ESTIMATIVA DA IDADE DE SEGREGAÇÃO DO LENHO JUVENIL E ADULTO POR MEIO DE PARÂMETROS ANATÔMICOS PARA MADEIRA DE Luehea divaricata Mart.
}

\author{
ESTIMATING THE AGE OF DEMARCATION OF JUVENILE AND MATURE WOOD IN Luehea \\ divaricata Mart.
}

\author{
Darci Alberto Gatto ${ }^{1}$ Clovis Roberto Haselein ${ }^{2}$ Ediane Andréia Buligon ${ }^{3}$ Leandro Calegari ${ }^{4}$ \\ Diego Martins Stangerlin ${ }^{5}$ Leonardo da Silva Oliveira ${ }^{1}$ Elio José Santini ${ }^{2}$
}

\section{RESUMO}

Este estudo teve como objetivo delimitar a idade de maturação do lenho usando-se de características anatômicas da madeira de Luehea divaricata Mart. (açoita-cavalo), por meio da segregação dos lenhos juvenil e adulto. Para tanto, foram eleitas três árvores adultas, de fuste reto e cilíndrico, com diâmetro superior a $30 \mathrm{~cm}$, a 1,30 m de altura (DAP), coletadas na Encosta Superior do Nordeste do estado do Rio Grande do Sul, Brasil. De cada árvore, a 0,10 m de altura da base do tronco, foi extraído um disco com aproximadamente $2 \mathrm{~cm}$ de espessura. Com auxílio de uma serra de fita, retirou-se de cada disco uma bagueta central, de $2 \mathrm{~cm}$ de largura, bem orientada no sentido radial, incluindo a medula no centro desta, dividindo-se a medula em duas amostras. Dentre as amostras sorteou-se uma e separou-se apenas o lenho inicial de cada anel de crescimento para a maceração (método de Jeffrey). Com auxílio de um microscópio esteroscópio equipado com régua micrométrica mediram-se primeiramente as dimensões individuais relativas ao comprimento, diâmetro e diâmetro do lume de cem fibras no anel de crescimento próximo à casca, e posteriormente, definiram-se trinta fibras, selecionadas ao acaso por anel de crescimento, como estatisticamente suficiente. A espessura da parede celular das fibras foi obtida pela metade da diferença entre os diâmetros da fibra e do lume. A segregação dos dois tipos de lenho foi avaliada com base na variação radial das características anatômicas (comprimento, diâmetro, diâmetro do lume e espessura da parede das fibras), por meio de regressão linear simples. Os resultados indicaram que, dentre as características estudadas, o comprimento de fibra foi a que melhor definiu o ano de segregação dos lenhos juvenil-adulto, estimado em 21 anos, aproximadamente. Por sua vez, os parâmetros anatômicos diâmetro das fibras, diâmetro do lume e espessura da parede das fibras mostraram-se inadequados para a estimativa da idade de segregação, devido aos baixos coeficientes de determinação dos modelos estatísticos testados.

Palavras-chave: lenhos juvenil e adulto; características anatômicas; idade de demarcação.

\section{ABSTRACT}

This study aimed at determining the age of demarcation of juvenile and mature wood of Luehea divaricata Mart., using anatomical characteristics. Three adult trees, in good trunk, with diameter at DBH larger than $30 \mathrm{~cm}$, from Encosta Superior do Nordeste do Estado do Rio Grande do Sul, were selected. Discs with thickness of $2 \mathrm{~cm}$ at $0.1 \mathrm{~m}$ of height from the base of the trunk were used. From each disc a radial ribbon $2 \mathrm{~cm}$ wide including the pith was removed. The initial wood of each growth ring was separated for maceration (method of Jeffrey). There were measured length and diameter of thirty fibers from each growth ring. Demarcation of the two types of wood was defined by the radial variation (pith-bark) of the anatomical characteristics (length, diameter, width of the lumen and wall thickness of fibers), using simple linear

1. Engenheiro Florestal, Dr., Professor Adjunto do Curso de Engenharia Industrial Madeireira, Universidade Federal de Pelotas - UFPel, Cx. Postal 354 - CEP 96019-900 Pelotas (RS). darcigatto@yahoo.com

2. Engenheiro Florestal, Dr., Professor Associado do Departamento de Ciências Florestais, Centro de Ciências Rurais Universidade Federal de Santa Maria Cidade Universitária "Prof. Mariano da Rocha Filho”, Av. Roraima, 1000, Bairro Camobi, CEP 97015-900, Santa Maria (RS). clovis.haselein@smail.ufsm.br

3. Acadêmica do Curso de Graduação em Engenharia Florestal, Centro de Ciências Rurais Universidade Federal de Santa Maria, Cidade Universitária "Prof. Mariano da Rocha Filho”, Av. Roraima, 1000, Bairro Camobi, CEP 97015-900, Santa Maria (RS). edianeandreia@yahoo.com.br

4. Engenheiro Florestal, MSc., Doutorando pelo Programa de Pós-Graduação em Ciência Florestal, Centro de Ciências Agrárias, Universidade Federal de Viçosa, Avenida P. H. Rolfs, s/n, CEP 36570-000, Viçosa (MG). leandrocalegari@yahoo.com.br

5. Engenheiro Florestal, Mestrando pelo Programa de Pós-Graduação em Engenharia Florestal, Centro de Ciências Rurais Universidade Federal de Santa Maria, Cidade Universitária "Prof. Mariano da Rocha Filho”, Av. Roraima, 1000, Bairro Camobi, CEP 97015-900, Santa Maria (RS).diego_stangerlin@yahoo.com.br

Recebido para publicação em 11/05/2007 e aceito em 6/10/2008. 
regression. Results indicate that fiber length is the best characteristic, while fiber diameter, lumen width and wall thickness were considered inadequate for demarcation. The age of demarcation of juvenile and mature wood of Luehea divaricata was defined as 21 years-old, approximately.

Keywords: juvenile and mature wood; anatomical characteristics; age of demarcation.

\section{INTRODUÇÃO}

O lenho juvenil ou madeira juvenil corresponde a uma região central da árvore, de forma cilíndrica, com diâmetro mais ou menos uniforme, estendendo-se desde a base até o topo da árvore, podendo formar parte do alburno ou do cerne no tronco, se esse último já estiver presente na árvore. Por sua vez, o lenho adulto ou madeira adulta é formado na fase adulta da árvore sempre posterior ao lenho juvenil (COWN, 1992). Para Ramsay e Briggs (1986), a madeira juvenil é o xilema secundário formado durante a fase juvenil do câmbio vascular da árvore (estágios iniciais da vida da árvore). Esse período varia conforme a espécie e sua proporção pode ser afetada pelas condições ambientais. A madeira juvenil caracteriza-se anatomicamente por um progressivo acréscimo nas dimensões das células e por correspondentes alterações na sua forma, estrutura e disposição em sucessivos anéis de crescimento.

A maioria dos estudos demonstram uma tendência de variabilidade radial do lenho, frequentemente descrita em zonas de madeira juvenil e adulta. A madeira juvenil é caracterizada por uma mudança progressiva nas características da célula e nas propriedades da madeira (PANSHIN e DE ZEEUW, 1970).

A variação radial aparece ao longo do tronco e pode ser explicada, em termos, por mudanças nas dimensões dos traqueídeos e, em último caso, pelo crescimento e produção hormonal (LARSON, 1973). Dessa forma, as propriedades da madeira não são uniformes no sentido medula-casca; pois nos primeiros anéis a madeira apresenta menor massa específica, menor comprimento de fibras, dentre outras características. Em anéis sucessivos, partindo do centro da árvore, verifica-se o aumento da massa específica e das características anatômicas (comprimento, diâmetro do lume e espessura das paredes das fibras etc.). A taxa de mudança na maioria das propriedades é muito rápida nos primeiros anéis; os anéis posteriores vão assumindo gradualmente as características da madeira adulta (BENDTSEN, 1978).

Embora a madeira juvenil não seja necessariamente inferior, por exemplo, para papel de imprensa e para a qualidade da impressão no papel (ZOBEL, 1984), é sabido que suas propriedades podem afetar algumas propriedades da madeira sólida. Dessa forma, há interesse considerável no seu conhecimento, pois a proporção desse tipo de lenho influencia na qualidade dos produtos de desbaste, no manejo e na administração da colheita final.

De modo geral, a maioria dos trabalhos sobre madeira juvenil enfatiza que o crescimento rápido nas plantações origina a formação de madeira de qualidade inferior e que, atualmente, é grande a proporção de madeira juvenil comercializada, trazendo como resultado problemas de qualidade nos produtos obtidos desse tipo de matéria-prima (BROWN e MCWILLIAMS, 1990). Assim, definir a idade aproximada em que ocorre a transição da madeira juvenil para madeira adulta, é de grande importância prática, já que a proporção de madeira juvenil no mercado é crescente. Essa informação permite estimar melhor os valores comuns entre as propriedades de madeira juvenil e adulta e, conseqüentemente, pode contribuir para uma melhor utilização da madeira juvenil e adulta (BENDTSEN e SENFT, 1986).

Dessa forma, para entender melhor a extensão e qualidade da madeira juvenil, precisa-se definir a demarcação entre os lenhos, uma tarefa difícil porque a mudança do lenho juvenil para lenho adulto, normalmente, ocorre gradualmente durante os anos (BENDTSEN, 1978). Para identificar a demarcação, já se utilizaram muitos métodos com grande complexidade e objetividade variada, desde identificar 0 aparecimento empírico da madeira juvenil, por exame visual, até análises complexas de seções transversais do tronco com aplicação de técnicas de regressão não-linear (ROOS et al., 1990). O método, freqüentemente, mais utilizado é o exame visual de desenhos gráficos das propriedades da madeira, em anéis observados a partir da medula, obtidos por meio de raios-X, maceração, entre outros (BENDTSEN e SENFT, 1986; CLARK e SAUCIER, 1989).

Shiokura (1982) definiu a idade de segregação da madeira por meio de uma fórmula logarítmica na qual descreveu a variação radial em comprimento de traqueídeos com o número do anel e a porcentagem de incremento anual em comprimento de traqueídeos. Neste, considerava que o ponto no qual o incremento anual em comprimento diminuiu $1 \%$, era o limite entre madeira juvenil e adulta. 
Já Loo et al. (1985), investigando a massa específica e o comprimento de traqueídeos em Pinus taeda L., utilizaram um procedimento de interação entre duas regressões lineares de dados de árvores individuais, uma para madeira juvenil e outra para madeira adulta, notando uma interseção entre elas. Cada repetição foi feita com os dois subconjuntos criados previamente, e a interseção da última repetição foi considerada como o limite entre madeira juvenil e adulta.

Objetivou-se com o presente trabalho delimitar a idade de maturação do lenho, usando-se características anatômicas da madeira de Luehea divaricata Mart. (açoita-cavalo), por meio da segregação dos lenhos juvenil e adulto.

\section{MATERIAL E MÉTODOS}

Três árvores adultas de Luehea divaricata Mart., com fuste reto e cilíndrico, em condições fitossanitárias adequadas e com diâmetro superior a $30 \mathrm{~cm}$ no DAP, foram coletadas em floresta nativa localizada na Encosta Superior do Nordeste do estado do Rio Grande do Sul, Brasil, extraídas em floresta nativa com licenciamento da Secretaria Estadual do Meio Ambiente.

O material foi selecionado pela extração ao acaso, conforme norma COPANT (1971) e ASTM D5536-94 (1995). De cada árvore retirou-se, a 0,10 m de altura desde a base do tronco, um disco com aproximadamente $2 \mathrm{~cm}$ de espessura.

Com o auxilio de uma serra-de-fita, retirou-se de cada disco uma bagueta central de $2 \mathrm{~cm}$ de largura, bem orientada no sentido radial, incluindo a medula no centro desta, a qual foi dividida na medula em amostras “A” e "B”, e armazenadas em solução de 70\% de álcool para manutenção de suas características. Dentre essas amostras, sorteou-se uma, da qual separou-se apenas o lenho inicial de cada anel de crescimento para a maceração e confecção de lâminas, segundo o método de Jeffrey descrito por Burger e Richter (1991).

Para estimar a idade ou demarcar a madeira juvenil/adulta, utilizou-se a medição de características anatômicas como comprimento, diâmetro, diâmetro de lume e espessura de parede das fibras. Primeiramente, mediram-se cem fibras no anel próximo à casca, e posteriormente, definiram-se trinta fibras por anel de crescimento como estatisticamente suficiente, medindo-se, o comprimento (C), diâmetro (D) e diâmetro do lume (d) de cada fibra (Figura 1). O comprimento das fibras foi medido em microscópio esteroscópio com régua micrométrica com aumento de dez vezes e o diâmetro das fibras e do lúmen, com aumento de quarenta vezes. A espessura (e) das paredes das fibras foi obtida pela metade da diferença do diâmetro das fibras e do lume (GATTO, 2006).

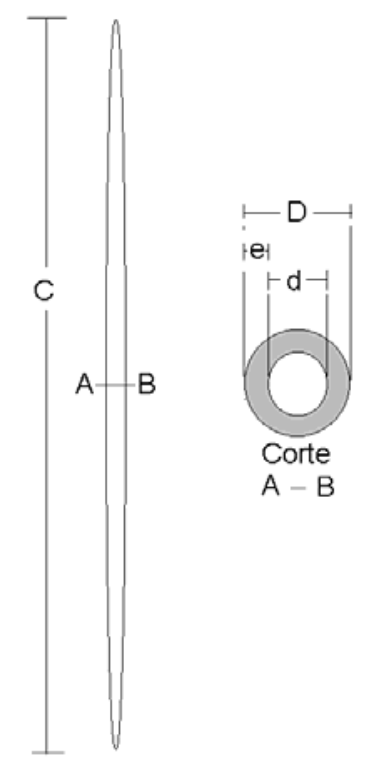

FIGURA 1: Esquema de medição das fibras ( $C$ = comprimento da fibra; $\mathrm{D}=$ diâmetro da fibra; $\mathrm{d}$ = diâmetro do lume; $\mathrm{e}=$ espessura da parede).

FIGURE 1: Details of fiber measurement $(C=$ length of the fiber; $D=$ diameter of the fiber; $d=$ diameter of the lumen; $\mathrm{e}=$ thickness of the wall).

A separação dos dois tipos de lenho foi definida pela variação radial (medula-casca) das 
características anatômicas (comprimento, diâmetro, diâmetro do lume e espessura de parede das fibras), através de duas regressões lineares simples. Aplicou-se uma regressão para a parte ascendente da nuvem de pontos e outra para a parte em que a nuvem se mostrava constante ou descendente. Os limites das duas nuvens de pontos foram definidos visualmente em gráfico antes do ajuste das equações. $\mathrm{O}$ ano de início de formação de lenho adulto foi determinado pelo cruzamento das duas retas obtidas pelas equações de regressão.

Para avaliar-se a segregação do lenho testaram-se equações lineares simples e quadráticas, bem como se utilizou a visualização de desenhos gráficos conforme Abdel-Gadir e Kramer (1993) e Clark e Saucier (1989). Optou-se pelas equações lineares simples, pois as quadráticas, nesse trabalho, apresentam dificuldades para delimitar, com precisão, o ano de segregação.

\section{RESULTADOS E DISCUSSÃO}

As Figuras 2 e 3 descrevem a variação do comprimento da fibra com a idade de formação da madeira de açoita-cavalo. Uma equação quadrática foi ajustada para o conjunto das fibras em função da idade (Figura 2). Apesar de significativo, o modelo explica apenas $38 \%$ da variação do comprimento da fibra em função da idade.

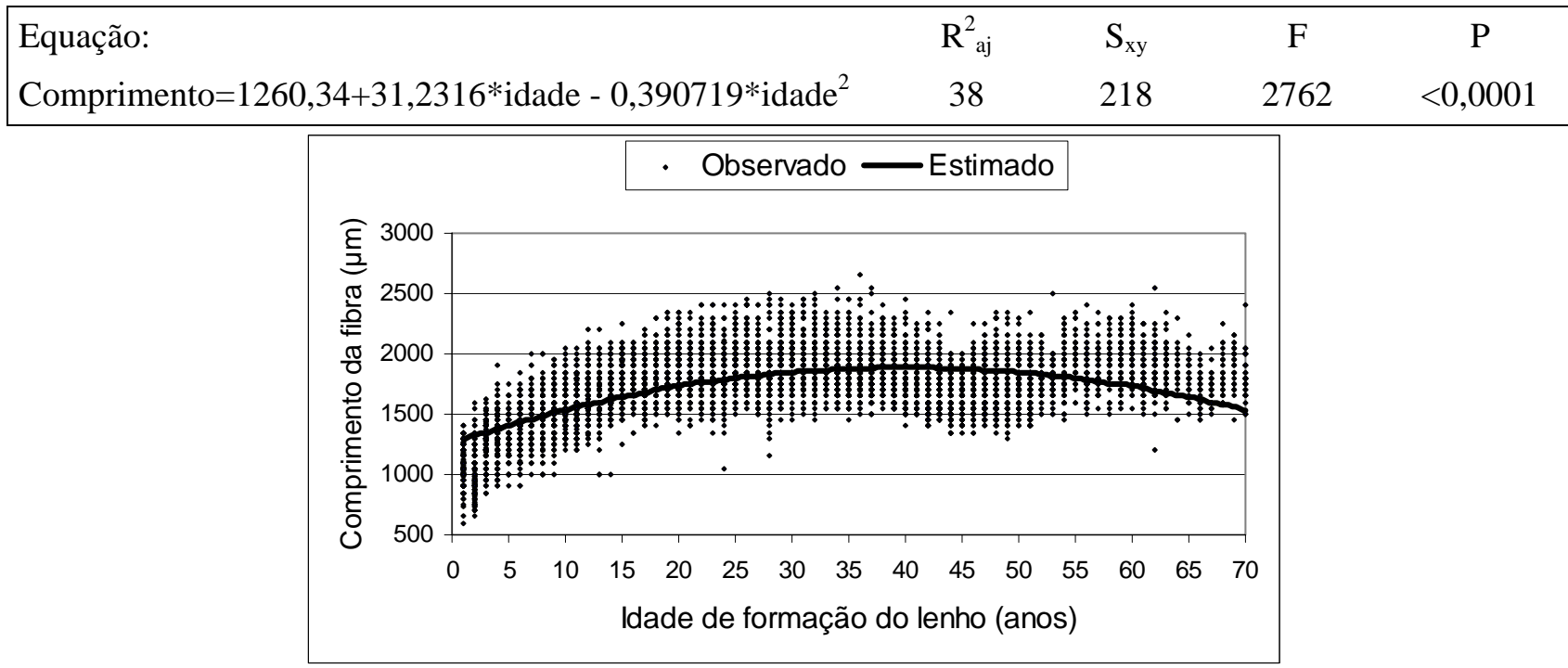

FIGURA 2: Variação do comprimento da fibra com a idade de formação do lenho para a madeira de Luehea divaricata para o conjunto de dados das três árvores estudadas.

FIGURE 2: Variation of fiber length of the wood of Luehea divaricata with age for the three studied trees.

A Figura 2 evidencia um aumento do comprimento das fibras, aproximadamente nos primeiros 20-30 anos de vida da árvore. A partir dessa idade o comprimento se estabiliza até ao redor dos 45 anos e, em seguida, oscila, diminuindo levemente. A variação radial do comprimento da fibra é grande, variando de extremamente curto $(<750 \mu \mathrm{m})$ nos primeiros anéis de crescimento (lenho juvenil) a extremamente longo $(>2000 \mu \mathrm{m})$ no lenho adulto.

Na Figura 3, com o auxílio de duas regressões simples, pode-se definir o ano de segregação dos lenhos juvenil-adulto em 21 anos aproximadamente, o que é levemente superior à faixa de segregação (5ํㅡo ao $20^{\circ}$ anel de crescimento) descrita para outras espécies pesquisadas (MUÑIZ, 1993; BENDTSEN, 1978).

Os parâmetros anatômicos diâmetro das fibras, diâmetro do lume e espessura da parede das fibras mostraram-se inadequados para a estimativa da idade de segregação entre o lenho juvenil e adulto, dado os baixos coeficientes de determinação. Para demonstrar a inadequação desses parâmetros na delimitação dos lenhos, nas Tabelas 1 e 2, são apresentadas as equações ajustadas para a madeira de açoita-cavalo. 


\begin{tabular}{|lcccc|}
\hline Equação: & $\mathrm{R}_{\text {aj }}^{2}$ & $\mathrm{~S}_{\mathrm{yx}}$ & $\mathrm{F}$ & $\mathrm{P}$ \\
a) Comprimento $=1158,54+34,8631 *$ idade & 62 & 190 & 6215 & $<0,0001$ \\
b) Comprimento $=1948,83-3,13954 *$ idade & 3 & 205 & 140 & $<0,0001$ \\
\hline
\end{tabular}

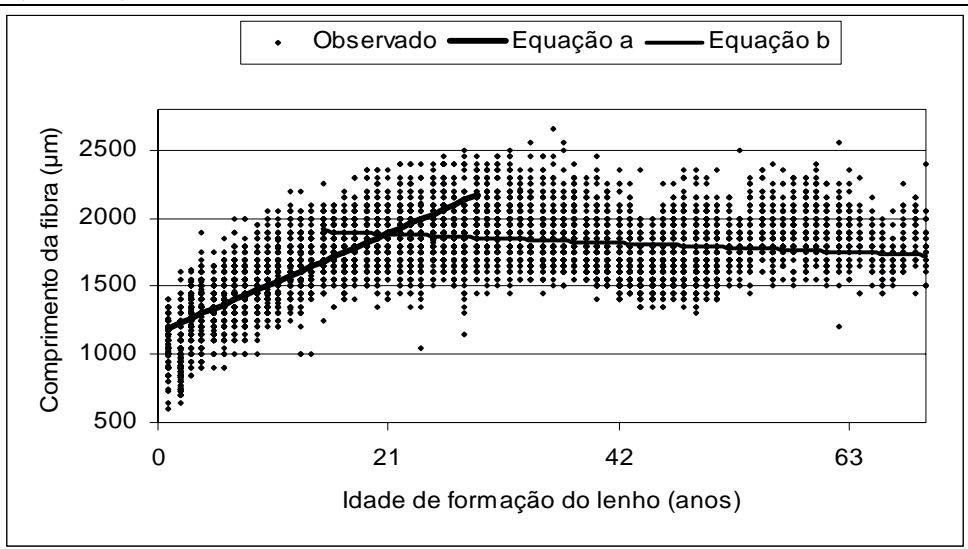

FIGURA 3: Idade de segregação do lenho juvenil e adulto em razão do comprimento da fibra da madeira de Luehea divaricata para o conjunto de dados das três árvores estudadas.

FIGURE 3: Age of demarcation of the juvenile and mature wood in relationship to the length of the fiber of the wood of Luehea divaricata for the three studied trees.

Verifica-se, na Tabela 1, que existe pouca relação entre as variáveis anatômicas e a idade. Em decorrência disso, o uso desses parâmetros para a segregação é impreciso, como pode ser visto na Tabela 2. Dessa forma, dentre as características anatômicas utilizadas neste estudo para estimar a idade mais provável de passagem do lenho juvenil para adulto, o comprimento das fibras foi a mais eficiente. Essa característica anatômica foi a que se apresentou mais adequada para avaliar a idade de segregação na direção radial da madeira. As demais características anatômicas, ou não foram influenciadas pela idade, ou apresentam uma diferenciação dimensional mais lenta e gradual, como também o afirmaram Muñiz (1993) e Bendtsen (1978).

TABELA 1: Equações ajustadas para estimar o diâmetro das fibras, diâmetro do lume e espessura da parede celular $(\mu \mathrm{m})$ para madeira de Luehea divaricata em função da idade.

TABLE 1: Adjusted equations to estimate the diameter of fibers, width of the lumen and thickness of the cell wall $(\mu \mathrm{m})$ for wood of Luehea divaricata considering its age.

\begin{tabular}{|c|c|c|c|c|}
\hline Equação & $\mathrm{R}_{\mathrm{aj} .}^{2}$ & $S_{y x}$ & $\mathrm{~F}$ & $\mathrm{P}$ \\
\hline Diâmetro fibras = 18,715+0,203242*idade-0,00305849*idade ${ }^{2}$ & 9 & 3 & 458 & $<0,0001$ \\
\hline Diâmetro lume $=10,9215+0,0689934 *$ idade-0,00107514* idade ${ }^{2}$ & 2 & 2,34 & 93 & $<0,0001$ \\
\hline Espessura parede $=3,8968+0,0671242 *$ anel-0,00099168* anel ${ }^{2}$ & 10 & 0,95 & 515 & $<0,0001$ \\
\hline
\end{tabular}

TABELA 2: Equações ajustadas para definição da idade de segregação do lenho juvenil (a) e adulto (b) em razão do diâmetro das fibras, diâmetro do lume e espessura da parede celular $(\mu \mathrm{m})$ para a madeira de Luehea divaricata.

TABLE 2: Equations adjusted for definition of the age of demarcation of juvenile (a) and mature (b) wood in reason of the diameter of fibres, width of the lumen and thickness of the cell wall $(\mu \mathrm{m})$ for the wood of Luehea divaricata.

\begin{tabular}{c|c|c|cc}
\hline Equação & $\mathrm{R}_{\text {aj. }}^{2}$ & $\mathrm{~S}_{\mathrm{yx}}$ & $\mathrm{F}$ & $\mathrm{P}$ \\
\hline a) Diâmetro fibras $=18,6351+0,156939 *$ anel & 5 & 2,81 & 111 & $<0,0001$ \\
b) Diâmetro fibras $=24,5635-0,0738317 *$ anel & 6 & 3,05 & 351 & $<0,0001$ \\
\hline a) Diâmetro lume $=10,7663+0,0653873 *$ anel & 3 & 2,44 & 132 & $<0,0001$ \\
b) Diâmetro lume $=12,8576-0,0262738 *$ anel & 2 & 2,28 & 114 & $<0,0001$ \\
\hline a) Espessura parede $=3,94244+0,0477642 *$ anel & 11 & 0,96 & 452 & $<0,0001$ \\
b) Espessura parede $=5,56541-0,00723665 *$ anel & 1 & 0,9 & 19 & $<0,0001$ \\
\hline
\end{tabular}




\section{CONCLUSÕES}

Analisando-se os resultados da variação radial das características anatômicas da madeira de açoita-cavalo pode-se concluir que:

O comprimento das fibras aumentou linearmente nos primeiros anos, seguindo-se de uma estabilização e de um pequeno decréscimo com o aumento da idade.

A segregação entre os lenhos juvenil e adulto pode ser determinada por meio do comprimento das fibras; com o auxílio de análise gráfica e modelos de regressão, estimou-se em 21 anos a idade de demarcação.

O uso do diâmetro das fibras, do lume e espessura da parede celular não permite definir claramente o ano de segregação dos lenhos juvenil e adulto do material estudado.

\section{REFERÊNCIAS BIBLIOGRÁFICAS}

ABDEL-GADIR, A.Y.; KRAHMER, R. L. Estimating the age of demarcation of juvenile and mature wood in Douglasfir. Wood and Fiber Science, v. 25, n. 3, p. 242-249, 1993.

ASTM. American Society for Testing and Materials. Standard practice for sampling forest trees for determination of clear wood properties: ASTM D5536-94. Philadelphia, PA: 1995.

BENDTSEN, B. A., Properties of wood from improved and intensively managed trees. Forest Products Journal, v. 28, n. 10, p. 69-72, 1978.

BENDTSEN, B. A.; J. SENFT. Mechanical and anatomical properties in individual growth rings of plantation-grown cottonwood and loblolly pine. Wood Fiber Science, v. 18, n. 1, p. 23-28. 1986.

BROWN, M. J.; McWILLIAMS, W. H. Pine stands across the South - trends and projections. In: SOUTHERN PLANTATION WOOD QUALITY WORKSHOP, 1989, Athens. Proceedings... Asheville: Southeastern Forest Experiment Station, United States Department of Agriculture, 1990. p. 1-15.

BURGER, L. M.; RICHTER, H. G. Anatomia da Madeira. São Paulo: Ed. Nobel, 1991. 154p.

CLARK, A.; SAUCIER, J. R. Influence of initial planting density, geographic location, and species on juvenile formation in souther pine. Forest Products Journal. V. 39, p. 42-48, 1989.

COPANT, Comision Panamericana de Normas Técnicas. Selección y colección de maderas, COPANT 30:1-001, 1971.

COWN, D. J. Corewood (Juvenile wood) in Pinus radiata - should we be concerned?. New Zealand Journal of Forestry Science, v. 22, n. 1, p. 87-95, 1992.

GATTO, D. A. Características tecnológicas do vergamento das madeiras de Luehea divaricata, Carya illinoinensis e Platanus $x$ acerifolia como subsídios para o manejo florestal. 2006. 109f. Tese (Doutorado em Engenharia Florestal) - Universidade Federal de Santa Maria, Santa Maria, 2006.

LARSON, P. R. The physiological basis for wood specific gravity in conifers. In: IUFRO Division 5 meeting, v. 2, Stellenbosch, 1973. Proceedings... Stellenbosch, 1973. p. 672-680.

LOO, J. A.; TAUER, C. G.; MCNEW, R. W. Genetic variation in the time of transition from juvenile to mature wood in loblolly pine (Pinus taeda L.). Silvae Genetetica, v. 34, n. 1, p. 14-19, 1985.

MUÑIZ, G. L. B. Caracterização e desenvolvimento de modelos para estimar as propriedades e o comportamento na secagem da madeira de Pinus taeda L.. 1993. 126f. Tese (Doutorado em Ciências Florestais) Universidade Federal do Paraná, Curitiba, 1993.

PANSHIN, A. J.; ZEEUW, C. de Textbook of wood technology. New York. Mc. Graw-hill. Compny, 1970. 105p.

RAMSAY, W.; BRIGGS, D. Juvenile wood: has it come of age?. In: A TECHNICAL WORKSHOP: JUVENILE WOOD-WHAT DOES IT MEAN TO FOREST MANAGEMENT AND FOREST PRODUCTS, 1985, Washington. Proceedings... Madison: Forest Products Research Society, 1986. p. 5-11.

ROOS, K. D.; SHOTTAFER, J. E.; SHEPARD, R. K., The relationship between selected mechanical properties and age in quaking aspen. Forest Products Journal, v. 40, n. 7/8, p. 54-56. 1990.

SHIOKURA, T.; Extent and differentiation of the juvenile wood zone in coniferous tree trunks. Wood Fiber Science, v. 28, n. 2, p. 85-90. 1982.

ZOBEL, B. J. The changing quality of the world wood supply. Wood Fiber Science, v. 18, p. 1-17. 1984. 